\title{
humanidades
}

Revista humanidades

Julio-Diciembre, 2015 •Volumen 5, número 2 • ISSN 2215-3934・pp. 1-22

\section{Un estudio acerca del cambio y el movimiento, a partir del pensamiento de Moisés Vincenzi}

DOI: http://dx.doi.org/10.15517/h.v5i2.21216

\section{Esteban Josué Beltrán Ulate}

Licenciado, estudiante de Maestría en la Escuela de Filosofía de la Universidad de Costa Rica. Funcionario del Ministerio de Cultura y Juventud en el Sistema Nacional de Educación Musical.

Correo electrónico: estebanbeltran@outlook.com

Más sobre el autor:

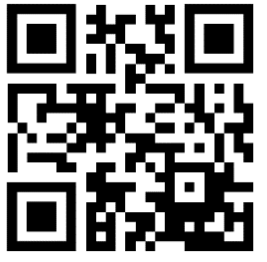

Todos los derechos reservados. Universidad de Costa Rica. Esta revista se encuentra licenciada con Creative Commons Reconocimiento-NoComercial-SinObraDerivada 3.0 Costa Rica. 


\section{Un estudio acerca del cambio y el movimiento, a partir del pensamiento de Moisés Vincenzi ${ }^{1}$}

\section{Resumen}

La pesquisa procura el reconocimiento de los aportes filosóficos de Moisés Vincenzi Pacheco. Específicamente, se analizan los considerandos vincenzianos sobre la no existencia del cambio y de la reducción al absurdo como herramienta metodológica para el estudio de las nociones de cambio y movimiento. Vincenzi logra despuntar al término de su reflexión una mirada contestataria a la concepción de sincronía y presenta un esbozo hacia una filosofía del infinitismo. Moisés Vincenzi, un filósofo imprudente ante la nada y testigo de la eternidad, el filósofo centroamericano más importante de los primeros decenios del siglo XX.
Palabras clave: Cambio, movimiento, eternidad, infinitismo, Vincenzi.

\section{A study of change and movement, from the thought of Moses Vincenzi}

\section{Abstract}

The research seeks recognition of the philosophical contributions of Moses Vincenzi Pacheco. Specifically, the article analyzes the vincenzianos thesis of nonexistence of change and contradiction as a methodological tool for the study of the notions of change and movement. Vincenzi manages to excel at the end of his reflection to a rebellious look conception of sync and provides an outline to a philosophy of infinitism. Moses Vincenci, philosopher reckless witness to nothing and eternity, the most important Central American philosopher of the early decades of the twentieth century.
Keywords: Change, movement, eternity, infinitism, Vincenzi. 
"Esa expresión, maestro, la nada y sus derivados, ¿es compleja?" (Moisés Vincenzi, Diálogos Filosóficos)

Moisés Vincenzi Pacheco (1895-1964) es uno de los filósofos más importantes en la primera parte del Siglo XX. ${ }^{2}$ Según Constantino Láscaris Comneno Micolaw, "Moisés Vicenzi Pacheco es... juzgando el conjunto de su obra impresa, el filósofo más maduro, completo y original que ha producido Centroamérica; y es ciertamente más estimable que muchos valores del continente que han gozado de más amplia caja de resonancia”. Así pues, este trabajo busca sumarse a los esfuerzos por reconocer los aportes filosóficos de dicho pensador en el ámbito costarricense y centroamericano.

La filosofía centroamericana adolece de proyección en el ámbito académico. En esta coyuntura es que emerge la tarea del presente estudio; mediar en el reconocimiento de la propuesta de Moisés Vincenzi en el ámbito de la Filosofía e Historia del pensamiento centroamericano.

La pesquisa acomete el análisis de las nociones de cambio y movimiento, según el filósofo costarricense, y una serie de derivaciones de su pénsum, en el que se perciben trazos de Parménides de Elea, Nietzsche, entre otros. ${ }^{3}$

Vincenzi decanta por la no existencia del cambio, lo que a la postre desencadena una serie de considerandos a propósito del cambio y movimiento, asumiendo como derrotero el principio de no contradicción y aplicando como herramienta metodológica la reducción al absurdo. 
Para el filósofo costarricense, asumir la existencia del cambio implica la aceptación del no-ser, en tanto que el cambio mismo supone el paso de ser algo a ser algo distinto del primer ser, ergo un algo-otro. Lo anterior permite colocar sobre la palestra el problema de la nada, ya que el paso de ser algo a ser otro algo, supone el dejar de ser del primer algo, algo que fue y ya no es, para asumir otro modo de ser en otro algo. Aunado a esto el nuevo ser, el otro algo, emergería ex nihilo, lo cual envuelve una problemática mayor.

Las tesis vincenzianas presentan una concepción de mundo plenista, planteamiento que deja sin lugar la posibilidad de vacíos, y por tanto de no-ser. El costarricense presumirá el cambio como un estado de conciencia de una totalidad diversa e infinita, "En el Universo todo es continuación de todo, todo es yo". ${ }^{4}$ Frente a lo anterior, asumirá el movimiento como la manifestación consiente respecto a la totalidad, "el movimiento es la conciencia de la diversidad". 5

A continuación, se busca presentar los considerandos vincenzianos y blandir una mirada crítica respecto a su razonamiento, procurando ante todo el reconocimiento del aporte filosófico del autor costarricense en el medio académico centroamericano. 
Moisés Vincenzi Pacheco nace en Tres Ríos de Cartago, un 3 de febrero de 1895, como comenta Víctor Rubio, en el texto intitulado "Estudio de un humanista: Vincenzi”. Es hijo del italiano Ceferino Vincenzi y de la costarricense Susana Pacheco Fernández. Moisés Vincenzi nunca fue un buen estudiante en primaria, a los doce años obtiene una beca para desarrollar estudios en el Liceo de Costa Rica; sin embargo, cinco meses después dilapidó su posibilidad de estudio al perder la subvención. Con apuros, ingresa a la Escuela Normal; no obstante, su retiro fue inminente dos años después, por bajas calificaciones.

Luego de este trance, Vincenzi se adentra en el mundo de la lectura, devorando descomunalmente libros, en franjas de 10 horas diarias de manera casi patológica. Se comenta que a los 17 años había adentrado en textos de Aristóteles y Nietzsche.

Con respecto a la educación superior, asiste a sesiones, como oyente, en la Facultad de Derecho y obtiene un Certificado Elemental de Pedagogía que le permite desempeñarse como profesor en Guanacaste. Posteriormente, retoma clases en la Escuela Normal de Heredia, donde se encuentra con el maestro Roberto Brenes Mesén, quien encuentra en el joven gran potencial; por lo que, procura guiarlo, de hecho, Brenes le llama "el joven filósofo".

Así las cosas, Moisés obtiene en 1916 el título de Maestro Normal.

Contrae nupcias en 1917, con Vitalina Pañaranda Campos. Se traslada a Heredia y alcanza la Dirección de la Escuela de San Rafael de Heredia. El filósofo cartaginés se licencia en Filosofía y Letras en la Universidad de Costa Rica, entre 1942-1948. 
Dentro de su haber académico, se desempeñó en la Escuela Normal de El Salvador (1936-1938), en el Instituto de Alajuela (1940-1944), en la Universidad de Costa Rica (1942-1949), en la Universidad Nacional de México y en la Real Universidad de León de los Caballeros, como doctor honoris causa. Huelga mencionar que desempeñó cargos en la Academia Costarricense de la Lengua, en la Dirección General de Bibliotecas Públicas (1945-1948) y en la Federación Costarricense de Fútbol. En 1962 obtiene en Costa Rica el Premio Magón.

En San José, un 22 de marzo de 1964, Don Moisés, aquel hombre "con su frente alta, ojos tristes, y cierta apariencia mediterránea", como el mismo se describió en una nota a la escritora costarricense de izquierda Carmen Lyra, fallece. Un día después, mediante el Acuerdo 587 de la Asamblea Legislativa costarricense, se declara Benemérito de la Patria. Esta acción, motivada por palabras como "Sin ribetes de exaltación lírica, podemos asegurar que el Prof. Vincenzi, cual padre amoroso, entregó frecuentemente a los jóvenes el fruto sazonado de su espíritu fecundo y dilecto", "En nuestro modesto mundo intelectual lleno de penumbras y suspicacia, Vincenzi ha adquirido ya una sólida posición de pensador profundo, vigoroso y renovador". ${ }^{6}$ En Guaycará de Golfito, en la provincia de Puntarenas, una Escuela lleva su nombre, el nombre del "joven filósofo", como diría Brenes Mesén. 
Respecto al pénsum filosófico vincenziano, se descubren distintas interpretaciones, el ex vicepresidente de Costa Rica, el intelectual Abelardo Bonilla Baldares, ${ }^{7}$ lo presenta como un pensador que decantó de manera general por el análisis de la razón y sus posibilidades cognoscentes, de las relaciones entre las nociones de forma y esencia y el carácter funcional de la palabra. En tanto que, desde la postura del filósofo costarricense Rodrigo Cordero $^{8}$, se concibe como parte de la agenda del pensador, la posibilidad retorno-eternidad en la obra de arte, las triadas Voluntad-SentimientoPensamiento y Arte-Ciencia-Filosofía, así como las categorías de Superhombre y Hombre máquina.

En esta misma línea descriptiva y caracterizadora, el filósofo Constantino Láscaris, concibe tres etapas en el pensamiento vincenziano. Un primer estadio (1915-1928) marcado por talento, pero a su vez por la inmadurez, en este momento destacan obras como "Mi Segunda Dimensión" y "Principios de la Crítica Filosófica". Posteriormente, un segundo momento (1930-1939) dirigido a la búsqueda de su postura filosófica, acá se descubren textos como "El caso Nietzsche", "El hombre máquina", "Marx en la Fragua". Y un tercer periodo, y momento culminante, donde se evidencia una suerte de sistematización de su pensamiento en su obra "El hombre y el cosmos. Síntesis de una filosofía" (1961).

El filósofo Láscaris, además, detalla a Vincenzi como aquel pensador que "se impuso la misión de la filosofía como información de la existencia concreta y esto le abrió el ámbito de la construcción abstracta del mundo, pero le polarizó también a la contracción racional del mundo". 9

\section{Aportes filosóficos de Vincenzi}


Moisés Vincenzi asume que el principal problema que ha de procurar resolver el filósofo, ha de ser el de su existencia. En ese sentido, a partir de las consideraciones que deriven de esta reflexión, debe meditar respecto a la continuidad de su yo, es así como esta problematización vacía sus interrogantes en la categoría de cambio. $\mathrm{El}$ autor cartaginés concibe como punto de partida el embate intelectivo de la noción de existencia y cambio, previo a cualquier acometida respecto a meditaciones en áreas de carácter trascendental.

Para llevar a cabo la tarea de elucidar el argumento de la existencia, se confronta la postura cartesiana del "je pense, donc je suis". ${ }^{10}$ Dicha confrontación se desprende de la consideración de mera evidencia, cuyo valor afirmativo es relativo. ${ }^{11} \mathrm{~A}$ propósito de la sentencia del parisino, el costarricense asume con toda firmeza una posición contestataria al criterio cartesiano e imputa a esta de ser una postura psíquica de alcances irresolutos: "La evidencia de su «pienso, luego soy» no pasó de ser un fenómeno psicológico tan indeterminable como otro cualquiera". ${ }^{12}$ Así pues, para Vincenzi no existe un sitio categórico sobre el cual la conciencia gire sobre sí, aceptar dicho giro implica la irrupción del proceso de relaciones del Yo consigo mismo y con la exterioridad; asumir una conciencia derramada sobre sí, es partir de galimatías.

\section{Del yo existo}

a la esencia

como

totalidad 
En contraposición a la visión de existencia fundada sobre la razón, como evidencia insoslayable e indubitable, cual fanatismo fruto de fenómeno evidencial, Vincenzi opta por esgrimir su argumento a tenor de la existencia del Yo. Dicha prueba, lejos de incrustar sus raíces en un argumento de carácter teológico, materialista o idealista, se erige teniendo como base el principio de no contradicción, y asumiendo como herramienta metodológica la Reductio ad absurdum.

Moisés Vincenzi argumenta la existencia del siguiente modo: "La manera única que tengo de declarar mi no existencia consiste en negarla. Pero para darle cuerpo a la negación es preciso que exista un poder que la declare. De manera, pues, que negar la existencia es poner en ejercicio un poder capaz de negar, lo cual es probar la existencia del ser que niega. Por lo tanto la existencia absoluta del ser no se puede negar en absoluto. Luego existo". ${ }^{13}$

La demostración vincenziana, esbozada atrás, manifiesta un olvido a la concepción cartesiana limitada en el cogito ${ }^{14}$ y se posiciona en un carácter analítico, tomando como herramienta metodológica la reducción al absurdo. Entonces, Vincenzi deja de lado la afirmación de la tesis (El Yo Existo) y decanta por asir de manera hipotética la negación de la tesis expuesta (Yo No-Existo), de lo cual se desprende un absurdo a partir de la concatenación de inferencias (Existencia de un ser que niega la existencia). Lo anterior permite concluir con rigor que la negación de la afirmación, que se había tomado como válida de manera hipotética, carece de razón para ser asumida (No se puede existir y no existir a la misma vez), esto consuma con la apropiación de la afirmación originaria (Ergo Existo). 
Insistiendo sobre la negación de la no-existencia, Vincenzi presenta la inconsistencia lógica de concebir el Yo y el No-Yo, ya que el No-Yo es la completa oposición del Yo, ambas nociones refieren antagonismos. Sin embargo, la totalidad es, por tanto no hay cabida para el No-Yo en la totalidad, desde esta óptica el universo comprendido como parte de la totalidad, es Yo, lo que permite derivar con claridad argumentativa que toda región del universo es continuidad de las demás. ${ }^{15}$

Lo anteriormente expuesto, si bien encuentra razón de ser en la demostración, que logra desbandarse de contradicciones lógicas, evidencia una serie de aspectos problemáticos, tales como el cambio y el movimiento, de lo existente en la totalidad, totalidad que precedentemente fue exhibida como continua continuidad.

La comprensión de cambio y movimiento vincenziana se desprende de la concepción de totalidad. Al respecto, el autor se apoya en la concepción de energía expuesta por la ciencia para referir a la realidad del cambio: "La ciencia contemporánea tiene probado que la energía es inmutable. Pero cree que existe una clase de cambio, el cambio de la forma que asume la energía al manifestarse"16. Lo anterior expresa que la energía sigue siendo energía, a pesar del modo de manifestación que asuma. En este contexto es que el autor presenta el planteamiento de Esencia, a partir de una lectura de las categorías de fenómeno y nóumeno.

\section{Cambio y movimiento en la continua continuidad de la totalidad}


Moisés Vincenzi adhiere a las categorías de fenómeno y nóumeno, como forma y esencia, esto no como una dualidad incompatible, sino más bien como una continuidad distinguible. El autor asiente en "aceptar la existencia de esa dualidad como la existencia de continuidad entre el espacio y lo inextenso". ${ }^{17} \mathrm{El}$ acogimiento de esta continuidad comprendida en dos talantes permite proferir una categoría englobante, de modo que el todo, la totalidad, es la esencia, así lo concibe el cartaginés: "De manera, pues, que todo es esencia. El cambio de forma, por consiguiente, debe ser una ilusión, o mejor dicho, un estado fijo de conciencia del yo infinito" ${ }^{18}$. Siendo así, el cambio es simplemente un relato imaginativo de la conciencia de la Esencia, me detengo a considerar que la conciencia no es asumida cual espectador frente a un escenario, sino más bien como actor que se descubre a sí mismo en el escenario.

La ciencia clásica, la oficial, en el contexto vincenziano, se mueve en la tradición del cambio, como el cambio de lugar de un ser, y de esta sentencia se desprende posteriormente el postulado de: "El ser que se mueve cambia en cuanto se mueve; pero como todo ser se compone de forma y esencia, en cuanto se mueve la esencia, cambia". ${ }^{19} \mathrm{Si}$ bien el mismo Vincenzi reconoce que la ciencia de su momento no afirma ni niega, con certeza, que la esencia de los seres, al moverse estos, se mueva, se permite problematizar ambas posibilidades, y de esta interpelación desboca en aporías. 
Si la esencia se mueve con los seres, implicaría que en el acto del movimiento tanto en forma como en esencia se darían cambios, siendo que A dejaría de ser A para converse en B, lo cual implicaría el cambio de lo inmutable, lo cual es absurdo. Si se asumiere como válida la concepción de que al moverse los cuerpos la esencia no lo hiciera, resultarían confusiones al intentar comprender como la Esencia permanece en un sitio luego del cambio mientras el accidente formal se encuentra en otro sitio, ${ }^{20} \mathrm{~A}$ pasa a ser B dejando parte de A fuera del ahora B.

Las posturas esgrimidas anteriormente, derivan en galimatías, puesto que en ambas el cambio supone dos situaciones. En primer término, el dejar de ser una cosa, como resultado del cambio que provoca que una cosa deje de ser algo que fue, siendo de este modo no-ser de aquello que dejo de ser en el proceso del cambio; y en segundo término, el ingresar a ser una cosa que no existía, lo cual implica una creatio ex nihilo, pues aquello que ahora es, no era, y su ser deriva del cambio. Evidentemente ambas posturas desbordan en contradicciones.

Frente a estas consideraciones, la tesis de cambio expuesta por Vincenzi resulta inquietante, pues se traduce como una manera distinta de comprensión del cambio y movimiento, en tanto que, en las antípodas de la visión tradicional, el pénsum vincenziano se libera de las contradicciones. 
En este escenario, el autor pondera la existencia de un fenómeno de cambio y movimiento basado en el reposo absoluto, por lo que todo aparente movimiento no es más que un reposo continuo e infinito, siendo que "la conciencia universal infinita de su diversidad infinita es la que se extiende a nuestros ojos la multiplicidad permanente de los cambios que creemos ver". ${ }^{21}$ De esta descripción expuesta por el autor, el movimiento se exhibe como conciencia de la diversidad de la esencia, es la contemplación de la sucesión de diferencia de los cuerpos.

La totalidad totalizante del universo como continua continuidad infinita, se manifiesta como siempre presente absoluto, cualquier consideración de cambio o movimiento no es más que la conciencia que se extiende sobre sí misma en la perpetua multiplicidad de la plenitud universal. Moverse por tanto es "sentirse en otros aspectos que son propiedad del ser infinito que se mueve en uno". En conclusión, para Moisés Vincenzi el cambio no existe y el movimiento se comprende como conciencia de la diversidad.

La tesis de universo infinito y fijo, le permite asumir la concepción de movimiento como conciencia de la prolongación del sí, en el ser infinito. De este modo, la única manera de aprobar el cese absoluto de movimiento sería a partir de la suspensión de la prolongación de sí, lo que evidentemente concluiría en una desnaturalización del absoluto. Por tanto, el autor asume que la evolución del intelectual del hombre, brota en el momento mismo del Yo que se asume como diverso en la infinitud. 
La cosmovisión vincenziana se caracteriza por mostrar un modelo de universo fijo, inmóvil, cual esencia referida como continuidad distinguible. De esta visión de mundo derivan; sin embargo, nuevas problematizaciones a la luz de los postulados de cambio y movimiento que se indicaron anteriormente, tal es el caso del cuerpo y el alma.

Vincenzi, a pesar de la distinción entre nociones, admite que ambas son necesarias e ineludibles una de otra, llegando incluso a calificarlas como "órganos uno del otro". De esta manera, el existente es una realidad fija, esto implica indubitablemente una declaración de inmortalidad tanto del cuerpo como del alma en su integridad.

La distinción de cuerpo y alma, ${ }^{22}$ es desarrollada en el pénsum vincenziano de un modo que permite responder a la aporía que deviene de pensar una existencia como unidad absoluta, ya que si este fuese el caso, esta unidad absoluta, cual mónada sin ventanas, no podría establecer relaciones ni con el exterior, ni consigo misma, lo cual no sería aceptable dentro de una visión de mundo en la que es posible el conocimiento. 
Según el filósofo, la relación solo es posible a partir de la analogía que procede de la multiplicidad, de este modo, la única manera posible para acceder a la relaciones con lo múltiple sería que la misma unidad absoluta sea, a su vez, diversa. Así, se advierte la capacidad de introspección del Yo y cómo de esta sobreviene el poder crítico que interviene en el modo de juzgar ${ }^{23}$ la exterioridad, su acceso al mundo del cual es parte, mediante un criterio. Con esto "queda comprobada la existencia de su divisibilidad, que entraña, naturalmente, la multiplicidad que se exige de la conformación del yo". ${ }^{24}$ En Vincenzi, la posibilidad de una comprensión de la existencia, a partir de nociones como Fenómeno y Noúmeno, queda resuelta en la visión integradora de la Esencia. ${ }^{25}$

Al igual que el alma y el cuerpo, el tiempo y el espacio son analizados por Vincenzi a la luz de su propuesta de universo fijo, pues según él, todo volumen admite dos dimensiones: tiempo y espacio. Así, mientras que en el

Tiempo, infinitud $\mathbf{y}$ esencia modelo euclidiano, las tres dimensiones se encuentran entre "dos nadas infinitas: el pasado y el porvenir", ${ }^{26}$ Vincenzi concibe que la tres dimensiones de Euclides ${ }^{27}$ subsisten en la dimensión del tiempo y espacio, estando las primeras subyugadas a las segundas: "En este sentido tanto podrá encontrarse en el tiempo como en el espacio la jerarquía tridimensional de Euclides". ${ }^{28}$

En el modelo del cartaginés, espacio y tiempo conforman "las dos dimensiones más características que conoce el hombre"29. La perspectiva del tiempo radica en la memoria, siendo la cercanía de relaciones con los acontecimientos lo que permite asumir algunos hechos en el pasado y 
otros en el presente. En lo que respecta al espacio, toda consideración deriva según la mayor o menor distancia del ámbito de operaciones de juicio, teniendo como referentes la relación entre el fenómeno cogitativo y el núcleo de facultades juzgado.

Para el autor, tanto la forma como la esencia son tiempo y espacio, forma y esencia son necesarias, y son necesariamente ser, por tanto inmutables. El filósofo costarricense acude a la visión de tiempo como eternidad, como tiempo imperecedero, como infinitud, esto implica una ruptura con la visión sincrónica del tiempo.

Vincenzi adhiere lo infinito como negación categórica de la Nada, esto permite comprender que en su infinitud lo infinito limite consigo mismo, ergo todo es infinito, esto a su vez conduce al esclarecimiento de límites entre esencia y forma, siendo así todo esencia, ya que como el mismo filósofo plantea "forma y esencia son la misma cosa en grados distintos", lo que le permite concluir afirmando "Todo es esencia en el Universo". ${ }^{1}$

Para el autor nada de la naturaleza desaparece, puesto que todo lo que nuestra conciencia nos muestra como pasado, presente e intuye como porvenir, resulta mera forma perecedera. Lo verdadero, según Vincenzi, es el tiempo Esencial, es inmutable es eterno, el "tiempo es la distancia potencial efectiva y objetiva que separa la sucesión de los actos, en virtud de la continuidad de lo diferente por lo

La eternidad, el infinitismo filosófico y desenlace análogo". ${ }^{32}$ En el modelo vincenziano, tanto el pasado, el presente y el porvenir, forman parte perpetua del presente universal, es por esta razón que en el hombre existe una capacidad por descubrir realidades del pasado y del porvenir. 
En esto radica lo que el autor denomina infinitismo filosófico, desde su planteamiento acrático, con el cual acomete una propuesta demoledora para abatir modelos antropocéntricos, que pretenden explicar la existencia, el universo, el cambio y el tiempo desde cogito, desde una perspectiva sensorial sesgada.

El filósofo oaxaqueño José María Albino Vasconcelos Calderón, en la presentación de la obra vincenziana intitulada Mi Segunda Dimensión, expresó "la figura de Vincenzi como uno de los más libres, penetrantes y atrevidos pensadores del Continente" ${ }^{\circledR 3}$, y no es en vano que se ha realizado dicha calificación, ya que de la propuesta teórica esgrimida en su primera etapa de pensamiento se desvelan una serie de considerandos problemáticos que se prestan para pesquisas en ámbitos especialistas. Si bien el abordaje de sus primeros escritos puede pecar de ser asistemáticos, en cada una de las consideraciones presentadas en su cosmovisión, se originan nuevos problemas por reflexionar, tales como el tema de la muerte, la concepción de Dios ${ }^{34}$, entre otros temas.

Moisés Vincenzi Pacheco, un pensador preclaro, figura trascendental en el pensamiento filosófico costarricense y centroamericano, un hombre que, como describe el historiador Enrique Mata Rivera, "era común verlo sentado en una banca del Parque Central de San José dialogando con sus amigos o paisanos, quienes se acercaban a nutrirse del verbo fácil y profundo de Vincenzi cuando se refería a los problemas morales y espirituales de una sociedad como la del siglo XX" ${ }^{35}$ Don Moisés, un hombre que se encuentra dialogando aún en San José, en el Salvador, en México y Nicaragua, filosofando en el eterno presente del universo esencial. 
1. Un avance de esta investigación se presentó como ponencia en

el IX Congreso Centroamericano de Filosofía, en la Universidad de Costa Rica, el miércoles 29 de octubre del 2014

2. Láscaris, Constantino. (1964). Desarrollo de las ideas filosóficas en Costa Rica. San José: Editorial Costa Rica.

3. Cordero, Rodrigo. (1975). Moisés Vincenzi. San José: Ediciones Ministerio de Cultura Juventud y Deportes.

4. Vincenzi, M. (1918). Principios de la Crítica, Roberto Brenes Mesén y sus Obras. Citado por Pacheco, Napoleón. (1920). En Filosofía de la Crítica, M. Vincenzi-Su personalidad crítica. San José: Imprenta Greñas.

5. Ídem.

6. Consejo de Redacción. (1963). Crónica, Revista de Filosofía, 4, (14). San José: Universidad de Costa Rica.

7. Bonilla, Abelardo. (1967). Historia de la literatura costarricense. San José: Editorial de Costa Rica.

8. Cordero, 1975

9. Láscaris, 1964, p.365

10. Descartes, 1637. Discurso del método.

11. Vincenzi, 1928. Principios de la Crítica Filosófica. Le livre libre. Paris; (PCF) 
12. Vincenzi, PCF, p. 14

13. Vincenzi, PCF, p. 67

14. Piedra, Rubén (1971). Gnoseología de Moisés Vincenzi. En Revista de Filosofía, 29, pp. 249-254. San José: Universidad de Costa Rica.

15. Vincenzi, PCF

16. Vincenzi, PCF, pp. 67-68

17. Vincenzi, PCF, p. 68

18. Vincenzi, PCF, p. p. 68

19. Vincenzi, M. (1928). Mi segunda dimensión. San José: Trejos.

20. Vincenzi, MSD.

21. Vincenzi, PCF, p.69

22. En otros momentos el Vincenzi recurre a las nociones de carne y espíritu, sobre esta segunda emite una caracterización tripartita, concibiendo el espíritu como afectivo, reflexivo y mixto. Vincenzi, PCF, p. 179

23. Para Vincenzi juzgar "no es conocer de modo absoluto la realidad de las cosas; es, simplemente, comparar y establecer grados de relación, partiendo de planos conocidos por sus relaciones comunes con el mundo ordinario". Vincenzi, PCF, p.96

24. Vincenzi, PCF, pp. 94-95 
25. Vincenzi, M. (1921). Diálogos Filosóficos. San José: Centro Intelectual Editor.

26. Vincenzi, MSD, p. 61

27. Las dimensiones expuestas por Euclides son tres: Largo, Ancho y Alto.

28. Vincenzi, M. (1927). América Libertada. San José: Trejos.

29. Vincenzi, M. (1920). Crítica Trascendental. San José: María V. de Lines.

30. Vincenzi, MSD, p. 41

31. Vincenzi, MSD, p. 42

32. Vincenzi, MSD, pp. 49

33. Vincenzi, MSD, p. 8

34. Al respecto, VIncenzi concibe a Dios como una necesidad, puesto que la realidad es demasiado densa para poder ser captada en la pequeñez del hombre.

35. Mata, 2010, p. 117 
Bonilla, Abelardo. (1967). Historia de la literatura costarricense. San José: Editorial Costa Rica.

\section{Referencias bibliográficas}

Consejo de Redacción. (1964). "Crónica”. Revista de Filosofía, 4 (14).

San José: Universidad de Costa Rica.

Cordero, Rodrigo. (1975). Moisés Vincenzi. San José: Ediciones

Ministerio de Cultura Juventud y Deportes.

Láscaris, Constantino. (1964). Desarrollo de las ideas filosóficas en Costa Rica. San José: Editorial Costa Rica.

Mata, Enrique. (2010). "Un pensador actuante ante la crisis moral generada por el hombre máquina”. En Revista Académica, 47. San José: Universidad Autónoma de Centroamérica.

Rubio, Víctor. (1941). Estudio de un humanista: Vincenzi. San Salvador: Nueva tribuna.

Pacheco, Napoleón. (1920). Filosofía de la Crítica, M. Vincenzi-Su personalidad crítica. San José: Imprenta Greñas.

Piedra, Rubén. (1971). Gnoseología de Moisés Vincenzi. En Revista de Filosofía, 29, pp. 249-254. San José: Universidad de Costa Rica.

Vincenzi, Moisés. (1920). Crítica Trascendental. San José: María V. de Lines.

Vincenzi, M. (1921). Diálogos Filosóficos. San José: Centro Intelectual. Vincenzi, Moisés (1927). América Libertada. San José: Trejos. 
Vincenzi, Moisés. (1928). Principios de la crítica filosófica. París: Le Livre libre.

Vincenzi, Moisés. (1928). Mi segunda dimensión. San José: Trejos.

Todos los derechos reservados. Universidad de Costa Rica. Esta revista se encuentra licenciada con Creative Commons Reconocimiento-NoComercial-SinObraDerivada 3.0 Costa Rica.

Correo electrónico: humanidades.eeg@ucr.ac.cr / Sitio web: http://revistas.ucr.ac.cr/index.php/humanidades 